\title{
Pituitary magnetic resonance imaging vs. bilateral inferior petrosal sinus sampling: comparison between non-invasive and invasive diagnostic techniques for Cushing's disease-a narrative review
}

\author{
Fabio Tortora ${ }^{1}$, Alberto Negro ${ }^{2}$, Francesco Briganti ${ }^{1}$, Maria Laura Del Basso De Caro ${ }^{3}$, \\ Luigi Maria Cavallo ${ }^{4}$, Domenico Solari ${ }^{4}$, Teresa Somma ${ }^{4}$, Luca Brunese ${ }^{5}$, Ferdinando Caranci ${ }^{6}{ }^{\wedge}$ \\ ${ }^{1}$ Unit of Interventional Neuroradiology, Department of Advanced Biomedical Sciences, "Federico II" University, Naples, Italy; ${ }^{2}$ Neuroradiology \\ Unit, Ospedale del Mare, Naples, Italy; ${ }^{3}$ Pathology Section, Department of Advanced Biomedical Sciences, "Federico II" University, Naples, Italy; \\ ${ }^{4}$ Division of Neurosurgery, Department of Neurosciences, Reproductive and Odontostomatological Sciences, "Federico II" University, Naples, Italy; \\ ${ }^{5}$ Department of Medicine and Health Sciences "V. Tiberio", University of Molise, Campobasso, Italy; ${ }^{6}$ Department of Precision Medicine, School of \\ Medicine "Luigi Vanvitelli", University of Campania, Naples, Italy \\ Contributions: (I) Conception and design: F Tortora, A Negro, F Caranci; (II) Administrative support: F Tortora, A Negro, F Caranci; (III) Provision \\ of study materials or patients: F Tortora; (IV) Collection and assembly of data: F Tortora, A Negro, F Caranci; (V) Data analysis and interpretation: F \\ Tortora, A Negro, F Caranci; (VI) Manuscript writing: All authors; (VII) Final approval of manuscript: All authors. \\ Correspondence to: Prof. Ferdinando Caranci, MD, PhD. Dipartimento di Medicina di Precisione, Università degli Studi della Campania Luigi \\ Vanvitelli, Naples, Italy. Email: ferdinando.caranci@unimol.it.
}

\begin{abstract}
Cushing's syndrome is a pathological clinical condition caused by an exposure of elevated cortisol levels over a long period of time. It is therefore essential to establish what the cause of hypercortisolism is. In most cases (about 80\%) the pathological process is due to adrenocorticotropic hormone (ACTH), while in a minor part of the cases (about 20\%) the cause is represented by a pathology of the adrenal glands and therefore not related to ACTH. Most patients with ACTH dependent Cushing's syndrome have a pituitary microadenoma; in the remaining cases (30\%), the high level of cortisol is linked to an ectopic secretion of ACTH. Surgical removal of the pituitary adenoma represents the treatment of choice in Cushing's disease (CD) patients; it is therefore necessary to identify and precisely locate the pituitary tumour responsible for the secretion of ACTH. Adequate diagnostic information is very often, even with magnetic resonance imaging (MRI), and in these cases we rely on bilateral inferior petrosal sinuses sampling (BIPSS). This procedure is considered the gold standard method for the diagnosis, but like any other diagnostic method it is not free from erroneous results such as false positives or false negatives.
\end{abstract}

Keywords: Cushing's disease (CD); magnetic resonance imaging (MRI); bilateral inferior petrosal sinus samples; microcatheter

Submitted Jun 22, 2020. Accepted for publication Oct 15, 2020.

doi: 10.21037 /gs-20-654

View this article at: http://dx.doi.org/10.21037/gs-20-654

\section{Introduction}

Cushing's syndrome is a clinical condition resulting after long-standing exposure to elevated levels of glucocorticoids (1). One of the main diagnostic challenges is identifying the underlying cause of hypercortisolism. In approximately $80 \%$ of cases, the cause is an adrenocorticotropic hormone (ACTH)-dependent process; in approximately $20 \%$ of cases the hypercortisolism is related to primary adrenal causes (that is, ACTH

\footnotetext{
^ ORCID: 0000-0003-4381-8323.
} 
independent) (2). Most of the ACTH-dependent cases (approximately $70 \%$ of all cases) are due to a pituitary adenoma [Cushing's disease (CD)]; in the remaining cases, the hypercortisolism is secondary to an ectopic ACTH secretion $(1,3)$. Surgical removal of the pituitary adenoma is the treatment of choice in patients with CD. Thus, localization of the source of ACTH secretion is critical in guiding timely treatment decisions. Exposure to elevated cortisol levels over a long period of time causes a very serious clinical pathological syndrome represented by Cushing's syndrome (1). Therefore the main diagnostic challenge to reach the most suitable therapeutic solution is to discover the cause of hypercortisolism. Pituitary adenoma is responsible for CD in most cases (about $70 \%$ of all cases) while the ectopic secretion of ACTH represents the cause of hypercortisolism in the remaining part $(1,3)$. The treatment of choice is the surgical removal of adenoma. It is therefore necessary to locate and identify the source of ACTH secretion to plan the treatment. We present the following article in accordance with the NARRATIVE REVIEW reporting checklist (available at http://dx.doi. org/10.21037/gs-20-654).

\section{Magnetic resonance imaging and its rule in the diagnostic workup}

The diagnostic method of choice for the diagnosis of CD is currently represented by magnetic resonance imaging (MRI) $(4,5)$. In the case of the suspicion of an ACTH secreting pituitary tumour, it is advisable to use a specific MRI study both in clinical practice and in pharmacological trials. MRI examination must be performed with a scanner of at least 1.5 Tesla with the use of coronal and sagittal T1-weighted spin-echo (SE) sequences before and after intravenous administration of gadolinium contrast medium and with T2-weighted sequence on the coronal plane, in order to obtain an optimal diagnostic level. High spatial detail is necessary. Thin sections (with a thickness of $2-3 \mathrm{~mm}$ or smaller), a fine matrix size $(256 \times 512$ or more) and a small field of view (FOV) (6) become fundamental technical parameters. A FOV focused on the pituitary region must be used to detect a microadenoma because, with the same matrix, a larger FOV offers a lower diagnostic resolution. In fact, a pituitary study conducted with a $12 \mathrm{~cm} \times 12 \mathrm{~cm}$ FOV has a higher resolution than a study conducted with an $18 \mathrm{~cm} \times 18 \mathrm{~cm}$ FOV, for the same matrix (7). A repetition time (TR) in the range of 500$700 \mathrm{~ms}$ and an Echo Time (TE) in the range of 15-
25 ms represent other important technical parameters (6), although better results have been obtained with shorter TR (400 ms) and TE (10.3 ms) (7). The use of the dynamic technique, with multiple coronal sequences after intravenous gadolinium injection, allows to achieve high sensitivity and specificity. A pituitary lesion can be found with this technique in $96 \%$ of patients with a biochemical diagnosis of ACTH-dependent CD (8), while in suspected CD patients a pituitary lesion can be identified with a $50-60 \%$ higher diagnostic sensitivity rate than that reported in nondynamic MRI. Moreover other technical factors are sagittal and coronal $\mathrm{T} 1-\mathrm{w}$ fat saturated post gadolinium sequences in order to better delineate the pituitary lesions in the postoperative follow up (9). The diagnostic sensitivity of an MRI study is also affected by the dose of contrast medium. The recommended dose of paramagnetic contrast medium for most intracranial lesions is $0.1 \mathrm{mmol} / \mathrm{kg}(10)$, but a $50 \%$ reduction of the usual dose of contrast medium can be used without a significant loss of sensitivity as described in many studies performed both on pituitary tumour and in normal gland $(11,12)$. However, there are limited and unclear data (13-16). The signal-to-noise ratio is essential to ensure adequate diagnostic value of an MRI exam. It is calculated from the difference in signal strength between the area of interest and the background and determines the graininess of the image. The grainier the image, the lower the signal-to-noise ratio. We can obtain an increase in the signal-to-noise ratio with the use of a three-dimensional (3D) acquisition with very thin sections (up to $1 \mathrm{~mm}$ ) (17), as for example with the MRI technique based on the use of $3 \mathrm{D}$ spoiled gradient recalled acquisition in the steady state (SPGR) sequence. This sequence allows to obtain a higher soft tissue contrast than the T1-w SE technique. Spatial resolution in MRI can be increased by two methods: (I) acquiring more data and signal averaging but with a longer acquisition time and thus increasing possible motion artifacts; (II) increasing the intensity of the magnetic field (18). The 3 Tesla operating machines allow MRI exams to be performed with greater spatial resolution with reduced acquisition time thanks to the high MR signal and offer many advantages for the identification of pituitary tumors (19). In particular, the 3 Tesla MRI allows a better identification of the pituitary microadenomas and a greater anatomical detail for the study of the parasellar region, useful for evaluating possible invasion of the cavernous sinus $(20,21)$. This is made possible thanks to the use of $3 \mathrm{D}$ T1-w sequences with a reduced slice thickness (1$2 \mathrm{~mm}$ ), which allows to obtain high quality images while 
minimizing susceptibility artifacts in the sellar and parasellar regions $(22,23)$. However, high-field imaging is not free from artifacts. The base of the skull as sellar and parasellar regions are susceptible to artifacts due to the interface of soft tissues, air and bones, which can be reduced with the use of three-dimensional fast SE (FSE) techniques. Despite the high magnetic field intensity and the various technical measures to reduce the artifacts, the identification of the secreting ACTH pituitary adenomas is still very difficult. In fact, in several case series involving adult patients, only $40-50 \%$ of pituitary adenomas were detected $(24,25)$. The identification of a pituitary lesion especially if less than $5 \mathrm{~mm}$ does not mean, however, to have found a pituitary adenoma responsible for a clinical symptomatology since a part of the general population between $10 \%$ and $20 \%$ has a pituitary incidentaloma $(25,26)$. In a recent meta-analysis $(27)$, an overall prevalence of pituitary adenomas of $16.7 \%$ was found $(14.4 \%$ in autopsy studies and $22.5 \%$ in imaging studies). For this reason it is very important to prove with further clinical instrumental evidence the presence of a pathological secretion of ACTH by the pituitary gland.

\section{Inferior petrosal sinus sampling and its rule in the diagnostic workup}

In the event that the results of clinical, biochemical and radiologic tests are equivocal or discordant, bilateral sampling of the inferior petrosal sinuses (IPSS) must be performed to confirm the presence of a secreting ACTH pituitary adenoma (28). Bilateral IPSS was introduced for the first time in the early 1980s $(29,30)$. Subsequently, its use with and without corticotropin releasing hormone $(\mathrm{CRH})$ administration was described in order to solve problems of differential diagnosis of Cushing's syndrome $(31,32)$. In this technique the operator inserts the sheaths bilaterally through the femoral veins. They are then advanced through the internal jugular veins into the lower petrosal sinuses.

Blood samples are taken from each sinus, obtaining them simultaneously, from both central catheters and the peripheral vein, to be able to compare plasma ACTH levels with each other. Serial samples are taken before and after administration of CRH ( $1 \mathrm{mg} / \mathrm{kg}$ body weight). In CD, a central to peripheral (central/peripheral) ACTH gradient results from high levels of ACTH in the venous drainage from the pituitary gland, whereas in the case of an ectopic ACTH secretion gradient it is not identified. A baseline ratio of central/peripheral ACTH values of 2.0 or greater is indicative of $\mathrm{CD}$ in case of non-administration of CRH (33). Since ACTH secretion is episodic and sampling can miss the burst of ACTH secretion, $\mathrm{CRH}$ is used as a stimulating agent to increase sensitivity, by collecting ACTH plasma samples from both inferior petrosal sinuses and peripherally at intervals after CRH administration. A central/peripheral ACTH ratio of 3.0 or higher suggests CD (31). Most patients with ectopic ACTH secretion exhibit a central/peripheral ACTH ratio of less than 2.0 before and after CRH administration (33). To obtain a valid IPSS study, there must be correct cannulation of the inferior petrosal sinuses. For this reason, it is recommended to perform checks with digital subtraction angiography to verify the correct positioning of the catheter and to correctly evaluate the venous anatomy (Figure 1). IPSS has high accuracy and efficiency in the diagnosis of Cushing's syndrome in experienced institutions, allowing to reach, for example, a sensitivity of $96 \%$ and a specificity of $100 \%$ in a recent meta-analysis of 21 researches, while thanks to the CRH stimulation the sensitivity can increase to $100 \%(34,35)$. In another study (36) the sensitivity for IPSS was $82 \%$ while it was $48 \%$ for the high dose dexamethasone test and $70 \%$ for the CRH test. When compared with other imaging modalities, IPSS showed a high accuracy: in a study performed on 54 patients (37) the accuracy of IPSS was $88 \%$ while the accuracy of MRI was $54 \%$; in another study where 84 patients were enrolled, the sensitivity was $40 \%$ for CT (38,39), $50 \%$ for MRI and $90 \%$ for IPSS (40). The diagnostic accuracy of MRI in differentiating central and ectopic Cushing's syndrome was found to be $58 \%$ while the accuracy of IPSS was found to be $100 \%$ (35). Several studies showed, however, that the diagnostic accuracy is not absolute and both false-negatives (lack of a central/ peripheral gradient in patients with $\mathrm{CD}$ ) and false positives (presence of a central/peripheral gradient in patients with ectopic ACTH secretion) occur. False negatives may occur in $10-15 \%$ of patients, with a higher prevalence on baseline, unstimulated ACTH levels (41). These patients usually present unilateral or bilateral anatomical variants in the petrosal venous system. Originally four variants were classified (42): the most common variant (Type I), being the petrosal sinus draining directly into the internal jugular vein (Figure 2). This classification was further modified and the most recent version describes six variants, with emphasis on the petrosal-jugular junction $(43,44)$. All these variants result in a success rate of less than $100 \%$ of bilateral petrosal cannulation and sampling (45). In a large 

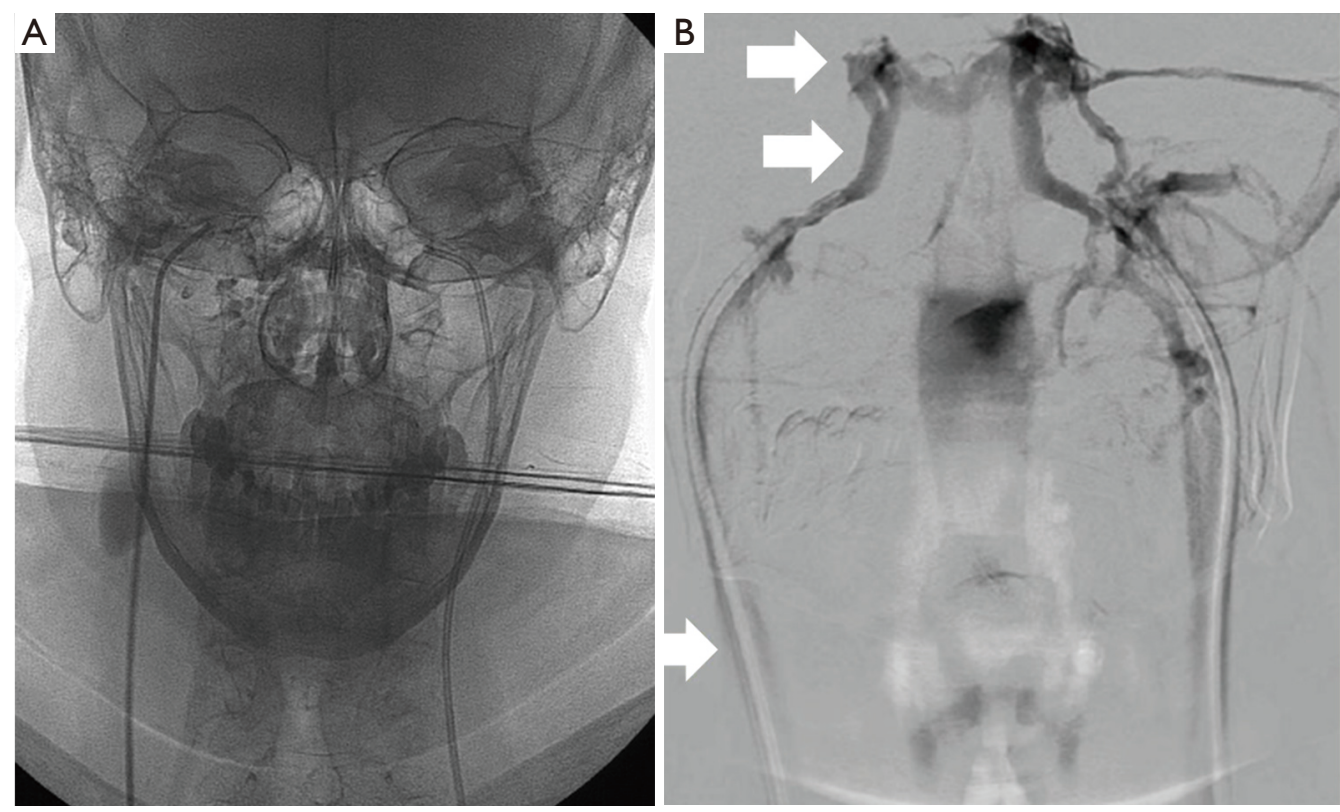

Figure 1 Inferior petrosal sinuses venography. (A) Well positioned catheters within the inferior petrosal sinuses. (B) Inferior petrosal sinuses venography during contrast medium injection. The white arrows indicate the inferior petrosal sinus draining directly into the internal jugular vein.
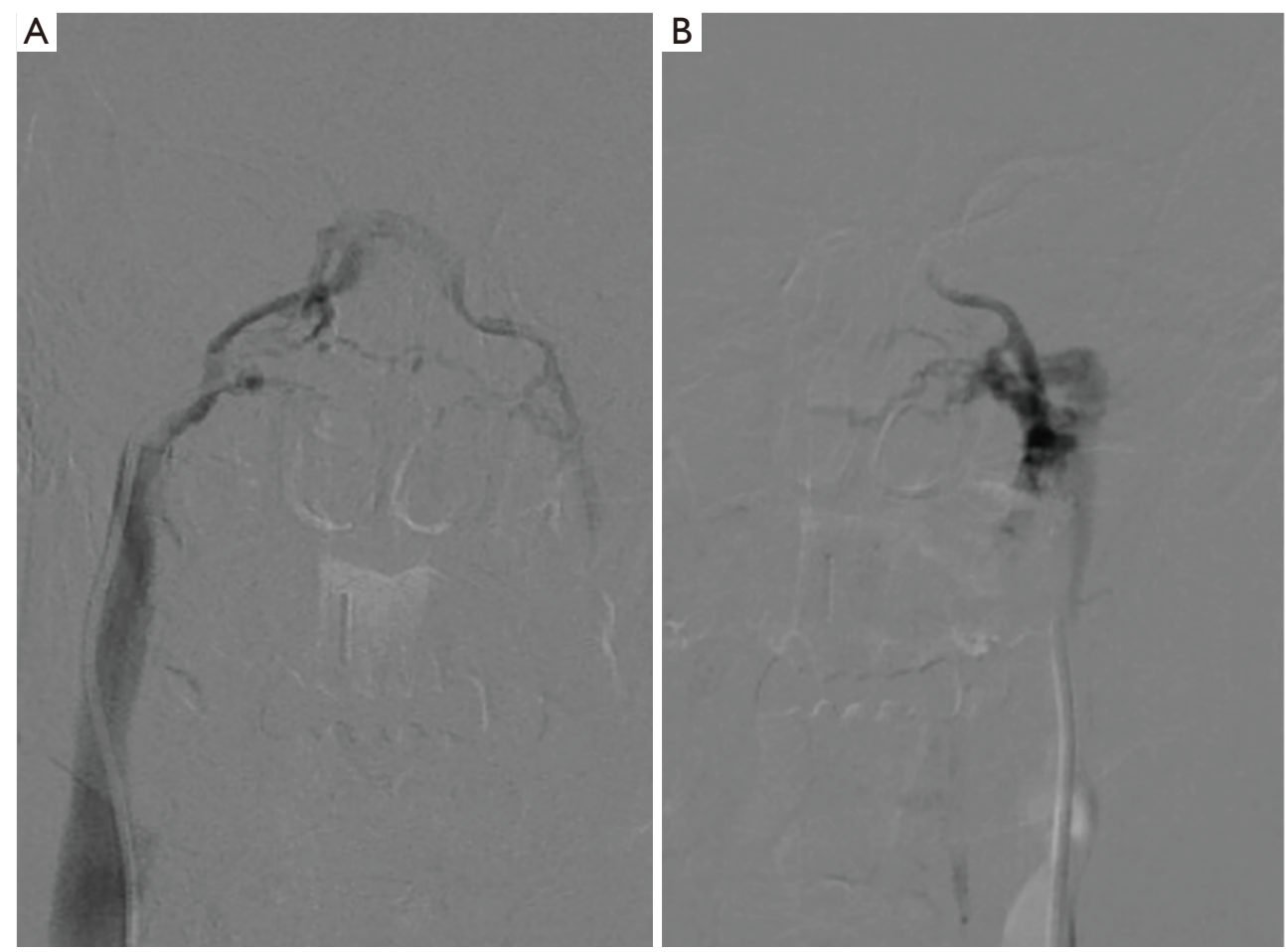

Figure 2 Patient with the initial diagnosis of Cushing's disease, in whom MRI showed no pituitary lesions. (A,B) Inferior petrosal sinus sampling found bilateral Type 1 drainage and a right-sided lateralization. Transsphenoidal right tumorectomy confirmed the presence of a pituitary adenoma. 
series (501 patients) (46) the false negatives obtained in $0.8 \%$ of the patients were due to a hypoplastic or abnormal inferior petrosal sinus. The variability in the tumoral corticotrope secretory pattern can also determine falsenegative results (45) and the secretion of ACTH by the adenoma in the petrosal sinuses at some time may not even reach a level 2 or 3 times higher than its peripheral serum concentration as usually expected. In patients with intermittent ectopic ACTH secretion there may be periods of time when cortisol levels are normal. Performing IPSS in such periods can lead to ambiguous results. False positives can also be caused by tumours that secrete $\mathrm{CRH}$ (47). Efforts to improve the diagnostic accuracy include additional sampling during IPSS for other anterior pituitary hormones, including prolactin for normalization of ACTH ratios. Since the inter-sinus ratio ACTH is known to be correct in less than $70 \%$ of cases, the intersinus ratio of prolactin has been used to identify the tumor site in the pituitary gland $(48,49)$. However, even with the use of this correct ACTH ratio for prolactin, the prediction of the tumour site sometimes remains uncertain (50). Higher diagnostic sensitivity may be achieved if IPSS is performed after stimulation with CRH. However, it is not readily available all over the world and then an alternative stimulating factor represented by desmopressin, more easily available, has been used $(51,52)$, obtaining excellent results over the years. About 150 patients with CD tested with desmopressin have so far been reported (53-55) showing a sensitivity comparable to that of stimulation with CRH (i.e., 90-97\%). The combined stimulation with the administration of both desmopressin and CRH together, performed to amplify the corticotropic responses, identified a central/peripheral ACTH gradient in 46 out of 47 patients with CD (56). However, the use of desmopressin is not free from serious complications since this agent is a known hemostatic and causes the release of von Willebrand factor and other factors of the coagulation cascade, which in a patient with CD could cause serious side effects (53). Bilateral IPSS is a reliable method if performed by an experienced interventional radiologist (57). The most common complication is the venous access site hematoma which can be seen in 3-4\% of patients (58). Serious complications are very rare. Pontine hemorrhage and vasovagal reactions as serious neurological complications have been reported in a large series of 508 patients. In the same series, minor and temporary complications such as dizziness and paraesthesia have been reported in three patients. In a different case report, the same authors reported medial medullar syndrome in a patient who developed non-hemorrhagic right medullary infarct site (58). Temporary 6th nerve palsy has been reported in two patients in a study of 86 patients (59). In a series of 94 patients, one patient suffered from venous subarachnoid hemorrhage resulting in acute obstructive hydrocephalus (60). In another series of 44 patients, brain stem infarct was detected by MRI in a patient (61). These complications are very rare and usually related to catheter selection and mapping or variations in venous anatomy. IPSS-related thromboembolic complications have also been reported. Since patients with Cushing's syndrome are predisposed to states of blood hypercoagulability, prophylactic heparinization during the procedure is very important $(62,63) ; 2$ of 43 patients were reported to develop deep vein thrombosis after IPSS, causing pulmonary thromboembolism in one of them; however, it was stated that no prophylactic heparinization was conducted (64). Deep vein thrombosis was documented in one of the 94 patients in another study, too (65). In our experience we have always subjected patients to prophylactic heparinization treatment without ever having encountered similar complications during and after the procedure. However, IPSS is a difficult procedure that requires technical mastery gained over the years. This led to look for simpler ways, such as jugular vein sampling. By comparing both methods, the sensitivity of jugular venous sampling was reported as $80 \%$ and IPSS as $95 \%(66)$. Another group reported the sensitivities of jugular venous sampling as $83 \%$ and IPSS as $94 \%$ (67). Although erroneous results need to be re-evaluated using IPSS, the jugular venous sampling can be used as an alternative procedure in institutions with limited technical experience, as we can read in these studies. An alternative to IPSS can be the cavernous sinus sampling (68). This technique would provide adequate and optimal central/peripheral ACTH gradients without even the administration of CRH since the cavernous sinuses are closer to the pituitary gland. It can be useful for identifying lateralization, but currently it is performed only by a limited number of neuroradiologists. During this procedure, technically similar to IPSS, the tip of the catheter is extended adjacent to the cavernous sinus. The position of the catheter is verified by venography of the cavernous sinus, performed by manual injection and with a minimal amount of contrast material in order to avoid complications, such as cerebral infarction and venous thrombosis. This superselective cavernous sinus sampling provided an accurate localization 
of the functional lesion in $73.3 \%$ without CRH stimulation and $93.3 \%$ with CRH stimulation $(66,68)$. Although this procedure can be considered an important additional tool in guiding lateralization its use is currently not routinary given its increased invasiveness and potentially increased risk of neurological complications (59).

Prospective studies with larger patient groups are required (58).

\section{Conclusions}

IPSS is an accurate and safe invasive diagnostic method in expert hands and plays an important role within the decisional algorithm for diagnosis and management of the Cushing syndrome.

\section{Acknowledgments}

Funding: None.

\section{Footnote}

Provenance and Peer Review: This article was commissioned by the Guest Editor (Antonio Barile) for the series "Multimodality Advanced Imaging and Intervention in Gland Diseases" published in Gland Surgery. The article has undergone external peer review.

Reporting Checklist: The authors have completed the NARRATIVE REVIEW reporting checklist. Available at http://dx.doi.org/10.21037/gs-20-654

Conflicts of Interest: All authors have completed the ICMJE uniform disclosure form (available at http://dx.doi. org/10.21037/gs-20-654). The series "Multimodality Advanced Imaging and Intervention in Gland Diseases" was commissioned by the editorial office without any funding or sponsorship. The authors have no other conflicts of interest to declare.

Ethical Statement: The authors are accountable for all aspects of the work in ensuring that questions related to the accuracy or integrity of any part of the work are appropriately investigated and resolved. This study does not require the approval from the ethics committee since the study is based on laboratory instrumental tests and on radiological examinations commonly used in the diagnosis of patients with Cushing's syndrome.
Open Access Statement: This is an Open Access article distributed in accordance with the Creative Commons Attribution-NonCommercial-NoDerivs 4.0 International License (CC BY-NC-ND 4.0), which permits the noncommercial replication and distribution of the article with the strict proviso that no changes or edits are made and the original work is properly cited (including links to both the formal publication through the relevant DOI and the license). See: https://creativecommons.org/licenses/by-nc-nd/4.0/.

\section{References}

1. Newell-Price J, Bertagna X, Grossman AB, et al. Cushing's syndrome. Lancet 2006;367:1605-17.

2. Bonelli FS, Huston J, Carpenter PC, et al. Adrenocorticotropic hormone-dependent Cushing's syndrome: sensitivity and specificity of inferior petrosal sinus sampling. AJNR Am J Neuroradiol 2000;21:690-6.

3. Nieman LK, Ilias I. Evaluation and treatment of Cushing's syndrome. Am J Med 2005;118:1340-6.

4. Colao A, Boscaro M, Ferone D, et al. Managing Cushing's disease: the state of the art. Endocrine 2014;47:9-20.

5. Escourolle H, Abecassis JP, Bertagna X, et al. Comparison of computerized tomography and magnetic resonance imaging for the examination of the pituitary gland in patients with Cushing's disease. Clin Endocrinol (Oxf) 1993;39:307-13.

6. Atlas S. Magnetic Resonance Imaging of the Brain and Spine. 4th edn. Lippincott, Williams and Wilkins, Philadelphia; 2009:1124-6.

7. Chowdhury IN, Sinaii N, Oldfield EH, et al. A change in pituitary magnetic resonance imaging protocol detects ACTH-secreting tumours in patients with previously negative results. Clin Endocrinol (Oxf) 2010;72:502-6.

8. Friedman TC, Zuckerbraun E, Lee ML, et al. Dynamic pituitary MRI has high sensitivity and specificity for the diagnosis of mild Cushing's syndrome and should be part of the initial workup. Horm Metab Res 2007;39:451-6.

9. Forbes K, Karis J, White WL. Imaging of the pituitary gland. Barrow Quarterly 2002;18:9-19.

10. Niendorf HP, Laniado M, Semmler W, et al. Dose administration of gadolinium-DTPA in MR imaging of intracranial tumors. Am J Neuroradiol 1987;8:803-15.

11. Davis PC, Gokhale KA, Joseph GJ, et al. Pituitary adenoma: correlation of half-dose gadolinium-enhanced MR imaging with surgical findings in 26 patients. Radiology 1991;180:779-84.

12. Giacometti AR, Joseph GJ, Peterson JE, et al. Comparison 
of full- and half-dose gadolinium-DTPA: MR imaging of the normal sella. Am J Neuroradiol 1993;14:123-7.

13. Bartynski WS, Boardman JF, Grahovac SZ. The effect of MR contrast medium dose on pituitary gland enhancement, microlesion enhancement and pituitary gland-to-lesion contrast conspicuity. Neuroradiology 2006;48:449-59.

14. Portocarrero-Ortiz L, Bonifacio-Delgadillo D, SotomayorGonzález A, et al. A modified protocol using half-dose gadolinium in dynamic 3-Tesla magnetic resonance imaging for detection of ACTH-secreting pituitary tumors. Pituitary 2010;13:230-5.

15. Splendiani A, Perri M, Marsecano C, et al. Effects of serial macrocyclic-based contrast materials gadoterate meglumine and gadobutrol administrations on gadoliniumrelated dentate nuclei signal increases in unenhanced T1weighted brain: a retrospective study in 158 multiple sclerosis (MS) patients. Radiol Med 2018;123:125-34.

16. Pasquini L, Rossi Espagnet MC, Napolitano A, et al. Dentate nucleus T1 hyperintensity: is it always gadolinium all that glitters? Radiol Med 2018;123:469-73.

17. Stadnik T, Stevenaert A, Beckers A, et al. Pituitary microadenomas: diagnosis with two-and three-dimensional MR imaging at $1.5 \mathrm{~T}$ before and after injection of gadolinium. Radiology 1990;176:419-28.

18. Shah S, Waldman AD, Mehta A. Advances in pituitary imaging technology and future prospects. Best Pract Res Clin Endocrinol Metab 2012;26:35-46.

19. Vitale G, Tortora F, Baldelli R, et al. Pituitary magnetic resonance imaging in Cushing's disease. Endocrine 2017;55:691-6.

20. Pinker K, Ba-Ssalamah A, Wolfsberger S, et al. The value of high-field MRI (3T) in the assessment of sellar lesions. Eur J Radiol 2005;54:327-34.

21. Wolfsberger S, Ba-Ssalamah A, Pinker K, et al. Application of three-tesla magnetic resonance imaging for diagnosis and surgery of sellar lesions. J Neurosurg 2004;100:278-86.

22. Kim LJ, Lekovic GP, White WL, et al. Preliminary experience with 3-Tesla MRI and Cushing's disease. Skull Base 2007; 17:273-7.

23. Raveendranath V, Nagarajan K, Umamageswari A, et al. Three-dimensional magnetic resonancebased morphometry of pituitary stalk. Radiol Med 2019;124:206-10.

24. Findling JW, Raff H. Screening and diagnosis of Cushing's syndrome. Endocrinol Metab Clin North Am 2005;34:385-402.
25. Hall WA, Luciano MG, Doppman JL, et al. Pituitary magnetic resonance imaging in normal human volunteers: occult adenomas in the general population. Ann Intern Med 1994;120:817-20.

26. Coulon G, Fellmann D, Arbez-Gindre F, et al. Latent pituitary adenoma. Autopsy study. Sem Hop 1983;59:2747-50.

27. Ezzat S, Asa SL, Couldwell WT, et al. The prevalence of pituitary adenomas: a systematic review. Cancer 2004;101:613-9.

28. Arnaldi G, Angeli A, Atkinson AB, et al. Diagnosis and complications of Cushing's syndrome: a consensus statement. J Clin Endocrinol Metab 2003;88:5593-602.

29. Oldfield EH, Chrousos GP, Schulte HM, et al. Preoperative lateralization of ACTH-secreting pituitary microadenomas by bilateral and simultaneous inferior petrosal venous sinus sampling. N Engl J Med 1985;312:100-3.

30. Oldfield EH, Girton ME, Doppman JL. Absence of intercavernous venous mixing: evidence supporting lateralization of pituitary microadenomas by venous sampling. J Clin Endocrinol Metab 1985;61:644-7.

31. Oldfield EH, Doppman JL, Nieman LK, et al. Petrosal sinus sampling with and without corticotropin-releasing hormone for the differential diagnosis of Cushing's syndrome. N Engl J Med 1991;325:897-905.

32. Giurazza F, Corvino F, Cavaglià E, et al. Arterial embolizations with microvascular plug in extracranial and intracranial districts: technical results. Radiol Med 2018;123:236-43.

33. Lad SP, Patil CG, Laws ER Jr, et al. The role of inferior petrosal sinus sampling in the diagnostic localization of Cushing's disease. Neurosurg Focus 2007;23:E2.

34. Newell-Price J, Trainer P, Besser M, et al. The diagnosis and differential diagnosis of Cushing's syndrome and pseudo-Cushing's states. Endocr Rev 1998;19:647-72.

35. Bekci T, Belet U, Soylu AI, et al. Efficiency of inferior petrosal sinus sampling in the diagnosis of Cushing's disease and comparison with magnetic resonance imaging. North Clin Istanb 2018;6:53-8.

36. Wiggam MI, Heaney AP, McIlrath EM, et al. Bilateral inferior petrosal sinus sampling in the differential diagnosis of adrenocorticotropin-dependent Cushing's syndrome: A comparison with other diagnostic tests. J Clin Endocrinol Metab 2000;85:1525-32.

37. Kaskarelis IS, Tsatalou EG, Benakis SV, et al. Bilateral inferior petrosal sinuses sampling in the routine investigation of Cushing's syndrome: A comparison with 
MRI. AJR Am J Roentgenol 2006;187:562-70.

38. Riccardi L, De Monte F, Cretti F, et al. Use of radiation dose index monitoring software in a multicenter environment for CT dose optimization. Radiol Med 2018;123:944-51.

39. Agostini A, Borgheresi A, Mari A, et al. Dual-energy CT: theoretical principles and clinical applications. Radiol Med 2019;124:1281-95.

40. Colao A, Faggiano A, Pivonello R, et al. Inferior petrosal sinus sampling in the differential diagnosis of Cushing's syndrome: Results of an Italian multicenter study. Eur J Endocrinol 2001;144:499-507.

41. Pecori Giraldi F, Cavallo LM, Tortora F, et al. The role of inferior petrosal sinus sampling in ACTH-dependent Cushing's syndrome: review and joint opinion statement by members of the Italian Society for Endocrinology, Italian Society for Neurosurgery, and Italian Society for Neuroradiology. Neurosurg Focus 2015;38:E5.

42. Shiu PC, Hanafee WN, Wilson GH, et al. Cavernous sinus venography. Am J Roentgenol Radium Ther Nucl Med 1968;104:57-62.

43. Mitsuhashi Y, Nishio A, Kawahara S, et al. Morphologic evaluation of the caudal end of the inferior petrosal sinus using 3D rotational venography. AJNR Am J Neuroradiol 2007;28:1179-84.

44. Gulmez Cakmak P, Ufuk F, Yagci AB, et al. Emissary veins prevalence and evaluation of the relationship between dural venous sinus anatomic variations with posterior fossa emissary veins: MR study. Radiol Med 2019;124:620-7.

45. Pecori Giraldi F, Pagliardini L, Cassarino MF, et al. Responses to corticotrophin-releasing hormone and dexamethasone in a large series of human adrenocorticotrophic hormone-secreting pituitary adenomas in vitro reveal manifold corticotroph tumoural phenotypes. J Neuroendocrinol 2011;23:1214-21.

46. Doppman JL, Chang R, Oldfield EH, et al. The hypoplastic inferior petrosal sinus: a potential source of false-negative results in petrosal sampling for Cushing's disease. J Clin Endocrinol Metab 1999;84:533-40.

47. Young J, Deneux C, Grino M, et al. Pitfall of petrosal sinus sampling in a Cushing's syndrome secondary to ectopic adrenocorticotropin-corticotropin releasing hormone (ACTH-CRH) secretion. J Clin Endocrinol Metab 1998;83:305-8.

48. Findling JW, Kehoe ME, Raff H. Identification of patients with Cushing's disease with negative pituitary adrenocorticotropin gradients during inferior petrosal sinus sampling: prolactin as an index of pituitary venous effluent. J Clin Endocrinol Metab 2004;89:6005-9.

49. Wind JJ, Lonser RR, Nieman LK, et al. The lateralization accuracy of inferior petrosal sinus sampling in 501 patients with Cushing's disease. J Clin Endocrinol Metab 2013;98:2285-93.

50. Mulligan GB, Faiman C, Gupta M, et al. Prolactin measurement during inferior petrosal sinus sampling improves the localization of pituitary adenomas in Cushing's disease. Clin Endocrinol (Oxf) 2012;77:268-74.

51. Moro M, Putignano P, Losa M, et al. The desmopressin test in the differential diagnosis between Cushing's disease and pseudo-Cushing states. J Clin Endocrinol Metab 2000;85:3569-74.

52. Reimondo G, Pia A, Bovio S, et al. Laboratory differentiation of Cushing's syndrome. Clin Chim Acta 2008;388:5-14.

53. Belli S, Oneto A, Mendaro E. Bilateral inferior petrosal sinus sampling in the differential diagnosis of ACTHdependent Cushing's syndrome. Rev Med Chil 2007;135:1095-102.

54. Castinetti F, Morange I, Dufour H, et al. Desmopressin test during petrosal sinus sampling: a valuable tool to discriminate pituitary or ectopic ACTH-dependent Cushing's syndrome. Eur J Endocrinol 2007;157:271-7.

55. Machado MC, de Sa SV, Domenice S, et al. The role of desmopressin in bilateral and simultaneous inferior petrosal sinus sampling for differential diagnosis of ACTH-dependent Cushing's syndrome. Clin Endocrinol (Oxf) 2007;66:136-42.

56. Tsagarakis S, Vassiliadi D, Kaskarelis IS, et al. The application of the combined corticotropin-releasing hormone plus desmopressin stimulation during petrosal sinus sampling is both sensitive and specific in differentiating patients with Cushing's disease from patients with the occult ectopic adrenocorticotropin syndrome. J Clin Endocrinol Metab 2007;92:2080-6.

57. Compagnone G, Padovani R, D'Avanzo MA, et al. Summary of the Italian inter-society recommendations for radiation protection optimization in interventional radiology. Radiol Med 2018;123:378-84.

58. Deipolyi A, Karaosmanoğlu A, Habito C, et al. The role of bilateral inferior petrosal sinus sampling in the diagnostic evaluation of Cushing syndrome. Diagn Interv Radiol 2012;18:132-8.

59. Lefournier V, Martinie M, Vasdev A, et al. Accuracy of bilateral inferior petrosal or cavernous sinuses sampling in predicting the lateralization of Cushing's disease pituitary microadenoma: Influence of catheter position 
and anatomy of venous drainage. J Clin Endocrinol Metab 2003;88:196-203.

60. Bonelli FS, Huston J 3rd, Meyer FB, et al. Venous subarachnoid hemorrhage after inferior petrosal sinus sampling for adrenocorticotropic hormone. AJNR Am J Neuroradiol 1999;20:306-7.

61. Gandhi CD, Meyer SA, Patel AB, et al. Neurologic complications of inferior petrosal sinus sampling. AJNR Am J Neuroradiol 2008;29:760-5.

62. Longo M, Bernava G, Calamuneri A, et al. MRI patient selection for endovascular thrombectomy in acute ischemic stroke: correlation between pretreatment diffusion weighted imaging and outcome scores. Radiol Med 2018;123:609-17.

63. Romano DG, Casseri T, Leonini S, et al. Single-center experience using the 3 MAX or 4 MAX reperfusion catheter for the treatment of acute ischemic stroke with distal arterial occlusions in patients not eligible for intravenous fibrinolysis. Radiol Med 2019;124:408-13.

64. Blevins LS Jr, Clark RV, Owens DS. Thromboembolic

Cite this article as: Tortora F, Negro A, Briganti F, Del Basso De Caro ML, Cavallo LM, Solari D, Somma T, Brunese L, Caranci F. Pituitary magnetic resonance imaging vs. bilateral inferior petrosal sinus sampling: comparison between non-invasive and invasive diagnostic techniques for Cushing's disease-a narrative review. Gland Surg 2020;9(6):2260-2268. doi: 10.21037 /gs-20-654 complications after inferior petrosal sinus sampling in patients with Cushing's syndrome. Endocr Pract 1998;4:365-7.

65. Swearingen B, Katznelson L, Miller K, et al. Diagnostic errors after inferior petrosal sinus sampling. J Clin Endocrinol Metab 2004;89:3752-63.

66. Doppman JL, Oldfield EH, Nieman LK. Bilateral sampling of the internal jugular vein to distinguish between mechanisms of adrenocorticotropic hormone-dependent Cushing syndrome. Ann Intern Med 1998;128:33-6.

67. Ilias I, Chang R, Pacak K, et al. Jugular venous sampling: An alternative to petrosal sinus sampling for the diagnostic evaluation of adrenocorticotropic hormonedependent Cushing's syndrome. J Clin Endocrinol Metab 2004;89:3795-800.

68. Fujimura M, Ikeda H, Takahashi A, et al. Diagnostic value of super-selective bilateral cavernous sinus sampling with hypothalamic stimulating hormone loading in patients with ACTH-producing pituitary adenoma. Neurol Res 2005;27:11-5. 